Check for updates

Cite this: J. Mater. Chem. A, 2019, 7, 21421

Received 5th July 2019

Accepted 5th August 2019

DOI: 10.1039/c9ta07269a

rsc.li/materials-a

\section{Low-tortuosity and graded lithium ion battery cathodes by ice templating $\dagger$}

\begin{abstract}
Chun Huang, (D) *a Martin Dontigny, ${ }^{\mathrm{b}}$ Karim Zaghib ${ }^{\mathrm{b}}$ and Patrick S. Grant ${ }^{\mathrm{a}}$
Preserving high energy densities of batteries at fast charge and discharge rates at the cell-stack level is a critical challenge for applications such as electric vehicles. Current manufacturing methods usually produce lithium (Li) ion battery electrodes $<100 \mu \mathrm{m}$ thin with unavoidable tortuous internal porosity that reduces energy densities at fast rates. Here, we use ice templating to manufacture ultra-thick $(900 \mu \mathrm{m})$ $\mathrm{LiFePO}_{4}$-based cathodes containing fast ion transport pathways and a pore structure gradient through the electrode thickness that promote high energy densities at fast rates. The electrodes exhibit $94 \mathrm{~mA} \mathrm{~h} \mathrm{~g}^{-1}$ at an ultra-high current density of $15 \mathrm{~mA} \mathrm{~cm}{ }^{-2}(67 \%$ higher gravimetric energy density at the cell-stack level including inactive components) compared with $47 \mathrm{~mA} \mathrm{~h} \mathrm{~g}^{-1}$ for conventional electrodes containing random structures and the same materials. X-ray computed tomography and modeling are used to quantify the electrode structure within different sub-domains and along orthogonal directions, which directly rationalizes the excellent dynamic performance. The electrode microstructure design, manufacturing method and characterization tools will be of use for other energy storage and conversion devices that rely on fast directional mass transport.
\end{abstract}

\section{Introduction}

To enable wider and faster adoption of lithium ion batteries (LIBs) in applications such as electric vehicles, the challenge of maintaining high energy density at high current (high power) must be overcome. ${ }^{1-3}$ A typical LIB cell comprises electrodes coated on metallic foil pieces (current collectors) that are stacked alternately (anode-cathode-anode-, etc.) with ion permeable porous separators in-between and wetted using a lithium (Li) ion containing liquid electrolyte., ${ }^{4,5}$ During charge and discharge, $\mathrm{Li}$ ions are transported through the electrode thickness and between the two electrodes. ${ }^{6}$ Most electrodes are made by a standard, highly productive slurry casting (SC) method involving coating a mixed slurry of active material particles onto the metallic foil followed by drying and calendering (pressing). One promising approach to increasing gravimetric and volumetric energy densities is to fabricate thick electrodes that reduce the proportion of inactive components (current collectors and separators) in cell-stacks. ${ }^{2}$ However, in practice, SC cannot be used to fabricate electrodes $>500 \mu \mathrm{m}$ thick without cracking or delamination from the metallic foil due to high internal strains and stresses accumulated by the large capillary forces generated during drying of the fugitive

${ }^{a}$ Department of Materials, University of Oxford, Oxford OX1 3PH, UK. E-mail: ann. huang@materials.ox.ac.uk

${ }^{b}$ Center of Excellence in Transportation Electrification and Energy Storage (CETEES), HydroQuebec, 1806 Lionel Boulet, Varennes, QC J3X1S1, Canada

$\dagger$ Electronic supplementary information (ESI) available. See DOI: 10.1039/c9ta07269a solvent. ${ }^{6-9}$ Additionally, SC produces random arrangements of the electrode materials with highly tortuous porosity that restricts effective ion transport (especially at fast charge and discharge rates), which in turn reduces the proportion of active material contributing to energy storage and capacities. ${ }^{10-13}$ Most current research studies focus on investigating new electrode materials and have made significant performance improvements. ${ }^{7,14-28}$ However, most of them use the ubiquitous SC route resulting in 40-60 $\mu \mathrm{m}$ thick electrodes.

Recently, there has been a growing interest in new processes that realise ultra-thick electrodes. For these electrodes to be competitive, the usual tortuous, random porosity of the electrodes must be replaced by a designed directionally porous structure. Examples include co-extrusion of a $\mathrm{LiCoO}_{2}$ (LCO) cathode ${ }^{29}$ magnetic templating of an LCO cathode, ${ }^{4,30}$ infiltration and carbonization of a natural wood framework to make an LCO cathode, ${ }^{31}$ and directional ice templating (DIT, or freeze casting) of a $\mathrm{LiNi}_{0.8} \mathrm{Co}_{0.15} \mathrm{Al}_{0.05} \mathrm{O}_{2}$ cathode ${ }^{32}$ and an $\mathrm{Fe}_{2} \mathrm{O}_{3}$ anode,$^{33}$ as well as other aligned pore structures for electrodes. ${ }^{34-36}$ We have previously reported LCO cathodes made by DIT with directional pores through the electrode thickness that showed a $\sim 20 \%$ increase in gravimetric capacity at $1 \mathrm{C}$ compared with electrodes of the same materials made by SC. ${ }^{37}$ The electrodes with directional and/or templated pores cannot be calendered (pressed) - a step for SC electrodes - because the electrode porous structure may be damaged. On the other hand, if performance can be achieved without the deformation process, this is an advantage that would allow elimination of a lengthy and expensive electrode processing step. However, 
these directional porous electrodes usually have a high pore volume (44-65 vol\% (ref. 30, 31, 33 and 37)) compared with the pore volume of conventional SC electrodes (30-40 vol\% (ref. 2)) which decreases energy densities. Additionally, none of the above studies have investigated a gradient pore structure which can further increase energy densities.

Here, we fabricate ultra-thick $(900 \mu \mathrm{m}) \mathrm{LiFePO}_{4}$ (LFP) cathodes with a low overall pore volume (36 vol\%) which still preserve low-tortuosity and pore structure gradient through the electrode thickness made by DIT. We investigate how this microstructure can improve the uniformity of dynamic ion distribution throughout the electrode and maintain high energy density at a high current. LFP is used as a model material with the advantages of good safety, high thermal stability, high tolerance to cycling and low cost. ${ }^{2,38}$ The DIT electrodes exhibit high capacities of 138 and $94 \mathrm{~mA} \mathrm{~h} \mathrm{~g}^{-1}$ at high current densities of 5 and $15 \mathrm{~mA} \mathrm{~cm}^{-2}$, respectively. X-ray computed tomography (XCT) and image-based modeling are used to quantify and understand how the 3D electrode microstructure affects Li ion transport kinetics in different sub-domains along orthogonal directions. Using our laboratory data, we calculate that for a constant volume LIB cell-stack including inactive components, the gravimetric and volumetric energy densities using the ultra-thick, low-tortuosity and graded DIT electrodes would significantly exceed those of the cell-stack containing standard SC electrodes.

\section{Results and discussion}

\section{Directional ice templating}

Fig. 1(a) and (b) show a schematic of the DIT apparatus and a temperature gradient $G v$ s. solidification rate $R$ diagram of the relationship between water freezing kinetics and the resulting ice structure, respectively. Fig. 1(a) shows that during DIT, a mixed aqueous-based slurry containing active LFP particles, electrically conducting carbon black nanoparticles and a binder is subjected to a steep temperature gradient along the direction normal to the sample plane imposed by a copper cold finger. Other solvents may also be used for DIT. ${ }^{39}$ Here, ice crystals are first nucleated on the surface of the cold finger at high (a)

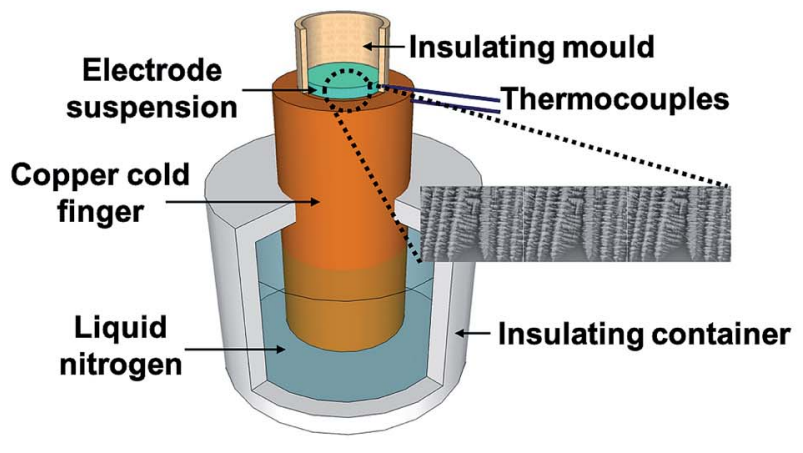

(c)

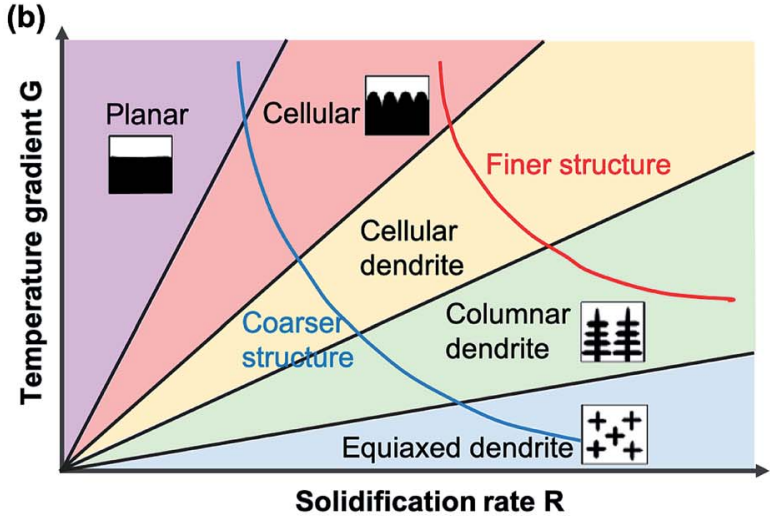

(ii) DIT LP-CC
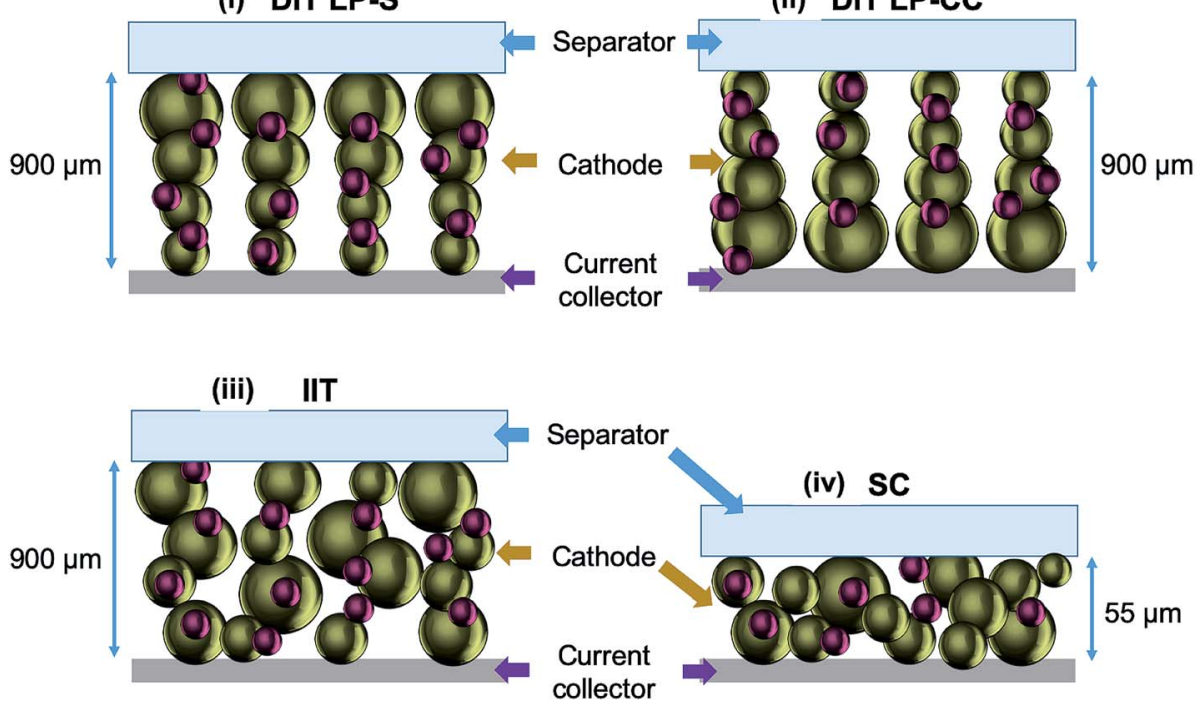

Fig. 1 (a) A schematic of directional ice templating (DIT); (b) a temperature gradient $G$ and solidification rate $R$ diagram showing how the final ice structure is controlled by the $G$ and $R$ conditions during the DIT process; and (c) schematics of the four electrode types fabricated for performance comparison: (i) directional ice templating with low porosity nearest the separator (DIT LP-S); (ii) directional ice templating with low porosity nearest the current collector (DIT LP-CC); (iii) isotropic ice templating (IIT); and (iv) slurry casting (SC). 
undercooling, leading to a high density of small crystals that are closely packed, i.e. low porosity and less elongated pores in this region after the ice is sublimed. As water continues to freeze, ice dendrites grow parallel to the temperature gradient and push the LFP and carbon black particles into the shrinking channels between the ice dendrites. These particles trapped in-between the ice dendrites are subjected to a small compressive force due to the volume expansion of water on freezing, and undergo a freeze-gelation process. ${ }^{40}$ The growing thickness of the frozen region with a high fraction of low thermal conductivity LFP progressively slows down the rate of heat extraction, leading to a transition from ice nucleation to ice growth, marked by a transition from cellular to columnar ice morphologies (Fig. 1(b)). ${ }^{41}$ The diameter of the elongated ice crystals also becomes progressively wider as cooling slows; ${ }^{40}$ thus an electrode structure with conical shaped elongated pore channels through the electrode thickness is produced. The ice crystals are then sublimed directly to vapour during freeze drying, leaving pore channels. Ice sublimation avoids strong capillary forces otherwise experienced in conventional liquid-vapour electrode drying processes, and so allows ultra-high thickness electrodes to be fabricated.$^{42}$ A slurry with a solid content of $\sim 40 \mathrm{vol} \%$ is used here (compared with the 8 to $30 \mathrm{vol} \%$ more usually used ${ }^{33,43}$ ) in an attempt to minimize final porosity while preserving the critical directional pore channels.

To understand the electrode microstructural effects on energy storage performance, we examine four types of electrodes in battery cells, all containing the same materials (Fig. 1(c)): (i) a $900 \mu \mathrm{m}$ thick, anisotropic structure made by DIT with low porosity near the separator (LP-S); (ii) a $900 \mu \mathrm{m}$ thick, anisotropic structure made by DIT with low porosity near the current collector (LP-CC); (iii) a $900 \mu \mathrm{m}$ thick, random structure made by isotropic ice templating (IIT), i.e. more uniform freezing in all directions; and (iv) a $55 \mu \mathrm{m}$ thick, random structure made by conventional slurry casting (SC) followed by calendering. The mass loadings of the four types of electrodes (60 samples per method) are summarized in Table 1. The mass loading of the SC electrodes is similar to that of the SC electrodes of the same materials in the literature. ${ }^{7}$ Due to the ultra-thickness, the mass loading of the ice templated electrode was $\sim 12$ times higher than that of the SC electrodes.

\section{Morphology}

Fig. 2(a) and (b) show scanning electron microscopy (SEM) images of the LFP particles that comprise micro-scale spherical secondary particles (Fig. 2(a)) formed from nano-scale primary particles (Fig. 2(b)), and primary and secondary particle size distributions are shown in Fig. S1 in the ESI. $\dagger$ Fig. S2 in the ESI $\uparrow$ shows an X-ray diffraction (XRD) pattern from an as-fabricated DIT electrode with all peaks corresponding to the $\mathrm{LiFePO}_{4}$ olivine structure. ${ }^{44}$ Fig. $2(\mathrm{c})$ shows an overview cross-sectional SEM image of the LFP-based DIT electrode with pores oriented approximately vertically through the electrode thickness. The electrode base (closer to the cold finger during DIT) contained smaller diameter $(\sim 500 \mathrm{~nm})$ pore channels, and the free surface contained larger diameter $(\sim 16 \mu \mathrm{m})$ pore channels, indicating an approximately conical pore channel shape consistent with a progressive transition from ice nucleation to cellular to columnar structures during the DIT process. Fig. 2(d) shows a higher magnification cross-sectional SEM image of the lower middle region of the DIT electrode in Fig. 2(c), with aligned "struts" of active material, which are further magnified in Fig. 2(e), indicating well-packed 85 to $760 \mathrm{~nm}$ thick struts corresponding to a single/a few layer(s) of primary particles. Fig. S3(a-c) in the ESI $\dagger$ show energy dispersive X-ray (EDX) maps of the electrode cross-sections confirming a uniform distribution of the active materials. Fig. 2(f) shows a cross-sectional SEM image of an LFP-based SC electrode, showing the distinctly different, typical random arrangement of particulates and tortuous pores. The particle agglomerate diameters were 10 to $24 \mu \mathrm{m}$, i.e. significantly coarser than those of the DIT electrode. Fig. S3 in the ESI $\dagger$ shows a cross-sectional SEM image of the IIT electrode, also showing a random arrangement of particulates and tortuous pores within a high electrode thickness. The random structures in Fig. $2(\mathrm{f})$ and $\mathrm{S} 4 \dagger$ suggest longer Li ion diffusion distances and relatively low electrode active material/ electrolyte contact areas. ${ }^{10,45}$

Table 1 A summary of properties for the four electrode types and their gravimetric and volumetric energy densities at the cell-stack level of the same total volume including inactive components of the separator and current collectors

\begin{tabular}{|c|c|c|c|c|}
\hline \multirow[b]{2}{*}{ Electrode } & \multirow{2}{*}{$\begin{array}{l}\text { Property } \\
\text { Mass loading }\left(\mathrm{mg} \mathrm{cm}^{-2}\right)\end{array}$} & \multirow{2}{*}{$\begin{array}{l}\text { Performance } \\
\begin{array}{l}\text { Current density } \\
\left.(\mathrm{mA} \mathrm{cm})^{-2}\right)\end{array}\end{array}$} & \multicolumn{2}{|c|}{ Energy density } \\
\hline & & & $\left(\mathrm{W} \mathrm{h} \mathrm{kg}^{-1}\right)$ & $\left(\mathrm{W} \mathrm{h} \mathrm{L}{ }^{-1}\right)$ \\
\hline \multirow{4}{*}{$\begin{array}{l}\text { (i) Directional ice templated, low porosity } \\
\text { near separator (DIT LP-S) } \\
\text { (ii) Directional ice templated, low porosity } \\
\text { near current collector (DIT LP-CC) } \\
\text { (iii) Isotropic ice templated (IIT) }\end{array}$} & \multirow[t]{2}{*}{$84.6 \pm 1.1$} & 5 & 408 & 411 \\
\hline & & 15 & 276 & 278 \\
\hline & $83.9 \pm 1.2$ & 5 & 351 & 354 \\
\hline & $87.1 \pm 2.0$ & 15 & 21 & 22 \\
\hline \multirow[t]{2}{*}{ (iv) Slurry casting (SC) } & \multirow[t]{2}{*}{$7.2 \pm 1.0$} & 5 & 245 & 388 \\
\hline & & 15 & 117 & 186 \\
\hline
\end{tabular}



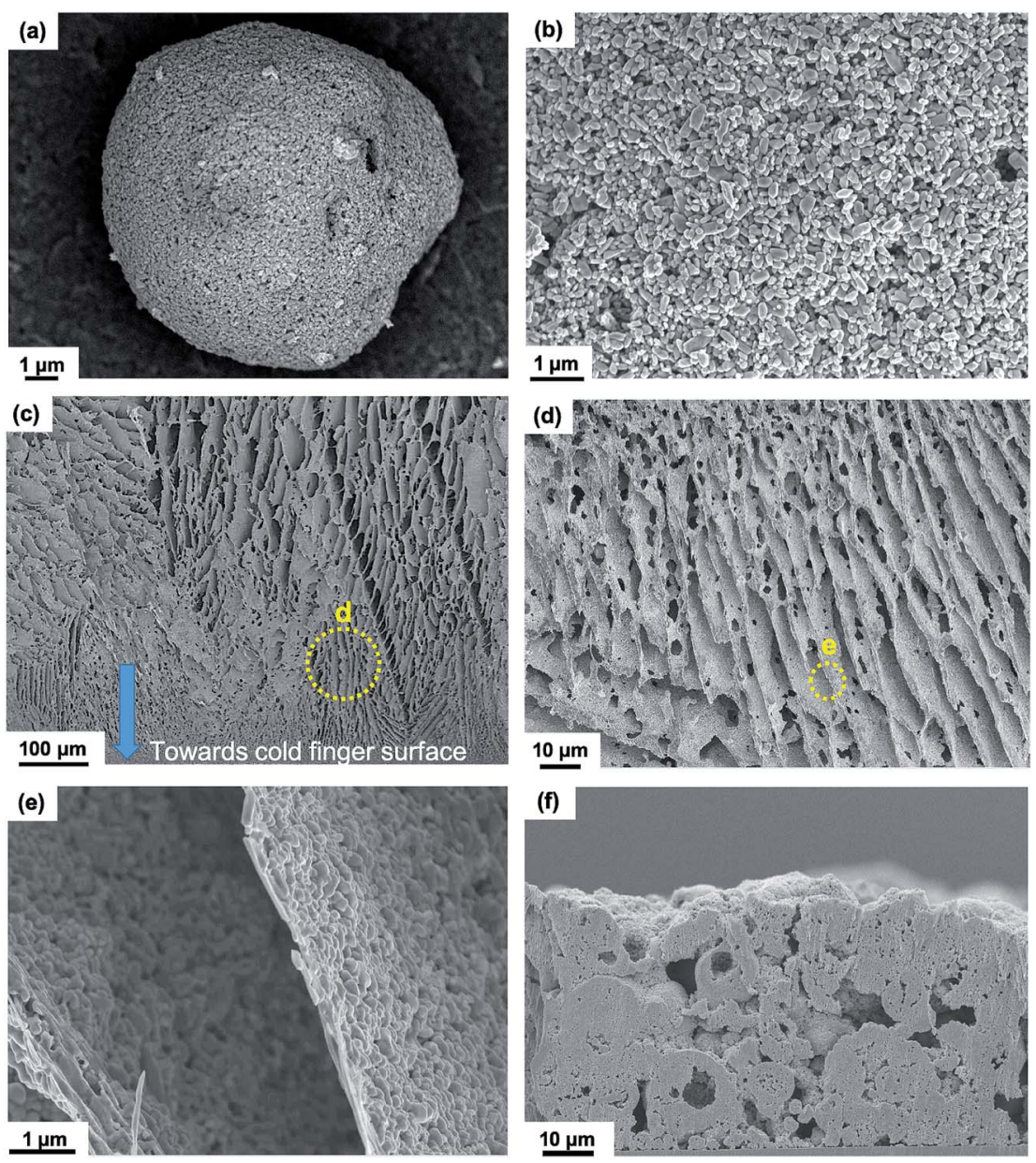

Fig. 2 SEM images of (a) LFP secondary particles and (b) LFP primary particles; (c) cross-sectional SEM image of a typical DIT electrode and its magnified regions (d) and (e); (f) cross-sectional SEM image of a typical SC electrode.

\section{Directional pore tortuosity and lithium ion diffusion simulations}

The definition of inter-connected pore tortuosity $\tau$ is the ratio of the distance an ion travels from one point to another through a twisted pore network to the straight-line distance between the same two points, where $\tau=1$ indicates an idealized straight path. ${ }^{46}$ Tortuosity is often used to quantify the difficulty with which ions can be transported by diffusive processes to all parts of the electrode. Experimentally, common methods of estimating $\tau$ such as mercury porosimetry provide a single overall tortuosity averaged over all possible directions of ion movement. ${ }^{47}$ These experimental approaches are not helpful for tortuosity characterization in microstructures with deliberate, anisotropic pore channels along one particular direction. In this case, pulsed field gradient nuclear magnetic resonance spectroscopy (PFG-NMR) along different directions may be employed to detect diffusion restriction inside the electrodes ${ }^{48,49}$ Here, we directly analyze the anisotropic tortuosity $\tau_{\text {direction }}$ and pore volume $\varepsilon$ in different locations and along orthogonal directions of the DIT electrode using X-ray computed tomography (XCT) and 3D image quantification with Li ion diffusion simulations. ${ }^{51}$
Fig. 3(a) shows a 3D volume rendered image of part of a DIT electrode comprising two discrete phases of the solid electrode materials (blue) and the pores (empty), where the $z$ direction is through the electrode thickness and the $x$ and $y$ directions are within the electrode plane. The overall pore volume of the DIT electrode was estimated to be $36 \mathrm{vol} \%$, similar to the $30-40 \mathrm{vol} \%$ porosity for the slurry cast electrodes, ${ }^{2,30}$ and lower than the $44 \mathrm{vol} \%$ in our previous work, ${ }^{37} 43 \mathrm{vol} \%$ for the electrodes made by magnetic alignment, ${ }^{30} 45$ to $55 \mathrm{vol} \%$ for the electrodes made by infiltration and carbonization of a wood framework, ${ }^{31}$ and 48 to $65 \mathrm{vol} \%$ for the other electrodes made by ice templating. ${ }^{33}$ The relatively low pore volume for the DIT electrode arose from minimizing the water fraction in the starting slurry compared with the solvent fractions used in other electrodes while maintaining viscosity and other rheological properties suitable for DIT. ${ }^{50}$ The 3D volume was then divided into 3 sub-domains: (i) further away from the cold finger surface during the DIT process, (ii) the middle of the electrode, and (iii) closer to the cold finger surface during the DIT process, and $\varepsilon, \tau_{x}, \tau_{y}$ and $\tau_{z}$ were estimated for each subdomain. The pore volume $\varepsilon$ was estimated to be 35,36 and $38 \mathrm{vol} \%$ in the bottom, middle and top sub-domains, indicating a slight porosity gradient through the electrode thickness. 


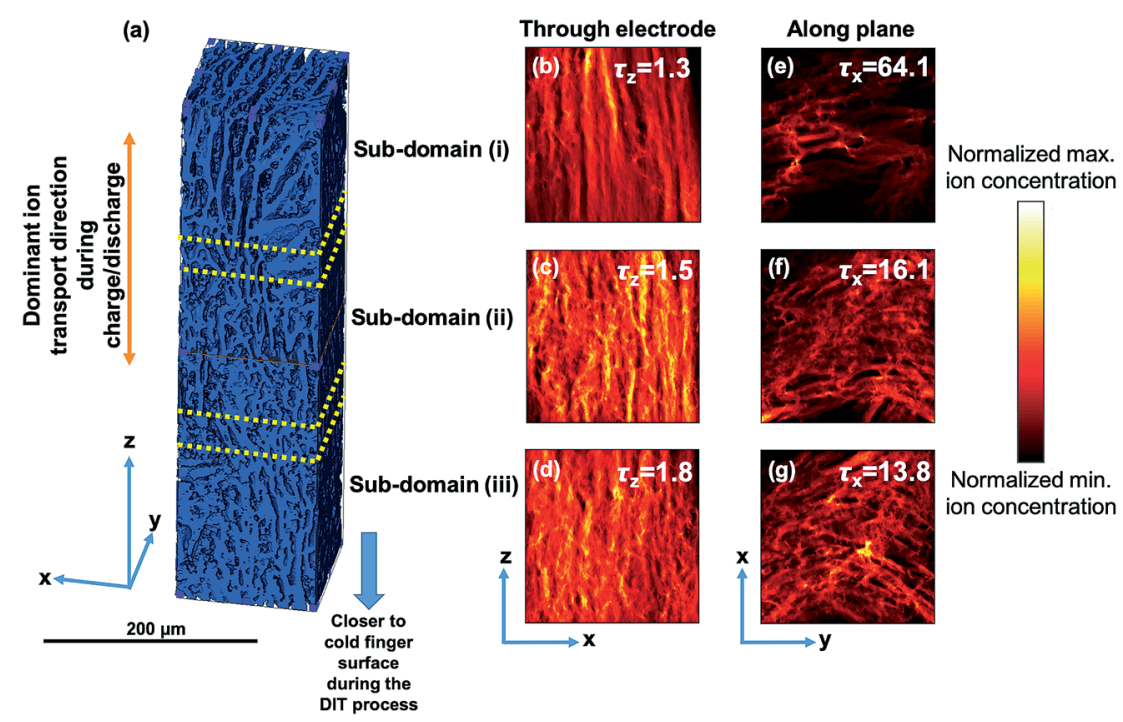

Fig. 3 (a) A 3D reconstruction of the anisotropic and graded electrode microstructure; (b-d) Li ion diffusion simulations in the $x-z$ plane with the relevant $\tau_{z}$ along the $z$ direction; and $(\mathrm{e}-\mathrm{g})$ Li ion diffusion simulations in the $x-y$ plane with the relevant $\tau_{x}$ along the $x$ direction, both for the subdomains (i) further away from cold finger surface, (ii) middle and (iii) closer to cold finger surface.

Finite difference method-based ion diffusion simulations were performed using the open source MatLab application TauFactor..$^{51}$ Briefly, the sub-domain was further divided into voxels and an ion concentration gradient was imposed between opposing faces of the sub-domain $(t=0)$. Ions were simulated to move through the structure through a plane to approach equilibrium $(t=x)$. Pore tortuosity $\tau$ was estimated by comparing the $\mathrm{Li}$ ion effective diffusive flux through the measured pore network $\left(F_{\mathrm{p}}\right)$ with the flux through an open volume of the same size $\left(F_{\mathrm{cv}}\right)$ using:

$$
\begin{gathered}
F_{\mathrm{p}}=-A D \frac{\varepsilon}{\tau} \frac{\Delta C}{L} \\
F_{\mathrm{cv}}=-A D \frac{\Delta C}{L}
\end{gathered}
$$

where $\varepsilon$ is the pore volume, $D$ is the diffusivity of $\mathrm{Li}$ ions in the electrolyte, and $\Delta C$ is the imposed, starting $\mathrm{Li}$ ion concentration difference across the sub-domain of thickness $L$ and crosssectional area $A$. Calculating the ratio of eqn (1) and (2) yields $\tau=F_{\mathrm{cv}} / F_{\mathrm{p}}$. This image quantification method does not account for electrochemical components as it does not apply a potential across the electrode. Fig. $3(\mathrm{~b}-\mathrm{d})$ and $(\mathrm{e}-\mathrm{g})$ show maps of the normalized, simulated ion concentration for each sub-domain in the $x-z$ and $x-y$ planes, respectively, demonstrating that from sub-domain (iii) (closer to the cold finger surface during the DIT process) to sub-domain (i) (further away from the cold finger surface during the DIT process), the ion transport pathways became increasingly aligned in the $z$ direction $\left(\tau_{z}=1.8\right.$ to 1.3) and more random in the $x$ direction $\left(\tau_{x}=13.8\right.$ to 64.1$)$. There was no significant difference in $\tau_{x}$ and $\tau_{y}$. Fig. S5 $\dagger$ shows how $\varepsilon, \tau_{z}$ and $\tau_{x}$ varied between sub-domains, indicating that $\varepsilon$ and $\tau_{z}$ had only small changes, but $\tau_{x}$ had significant increases through the electrode thickness, and $\tau_{x}$ was 7 to 48 times higher than $\tau_{z}$.
Since the spatial resolution of the lab source XCT was $700 \mathrm{~nm}$, the lab source XCT was more suited to characterizing the DIT electrodes, which contained the majority of aligned micro-pores compared to the IIT and SC electrodes which contained a wide pore size range from as small as $80 \mathrm{~nm}$ up to 7 $\mu \mathrm{m}$ (Fig. 2(f) and S3†). However, synchrotron XCT (with an improved voxel resolution of $20 \mathrm{~nm}$ ) investigations of commercial SC electrode structures showed $\varepsilon=37-41 \%$ and $\tau_{z}$ $=3.3-4.9,{ }^{46,52,53}$ demonstrating a $54-69 \%$ decrease in $\tau_{z}$ through the electrode thickness for the DIT electrode compared with these conventional SC electrodes.

A gradient porosity microstructure is typical of ice crystallization for the relatively thick DIT electrodes. Methods of maintaining a constant pore diameter and pore fraction have been investigated for templating-based processing methods, but achieving this while preserving scalability is difficult. ${ }^{54}$ Instead, we now consider how this gradient microstructure can be exploited. This would allow a judgement on the need for further efforts to either promote or suppress porosity and help to assess the scalability of the DIT processing. It was hypothesized that orienting the electrode with the region of low $\varepsilon$, low $\tau_{z}$ and low $\tau_{x}$ against the separator where ion concentration is high and with the region of higher $\varepsilon$, even lower $\tau_{z}$ and high $\tau_{x}$ against the current collector where ion diffusion is often restricted ((i) DIT LP-S, Fig. 1(c)) may obtain a better electrode dynamic response than the converse ((ii) DIT LP-CC, Fig. 1(c)), and these experiments are now described.

\section{Electrochemical properties}

Fig. 4(a) shows a cyclic voltammogram of the DIT (LP-S) electrode at a scan rate of $0.05 \mathrm{mV} \mathrm{s}^{-1}$. The peaks at $3.6 \mathrm{~V}$ during charge and at $3.3 \mathrm{~V}$ during discharge were associated with a typical two-phase transition between $\mathrm{LiFePO}_{4}$ and $\mathrm{FePO}_{4}$ during charge and discharge.$^{55} \mathrm{Fig} .4(\mathrm{~b})$ shows the galvanostatic 
(a)

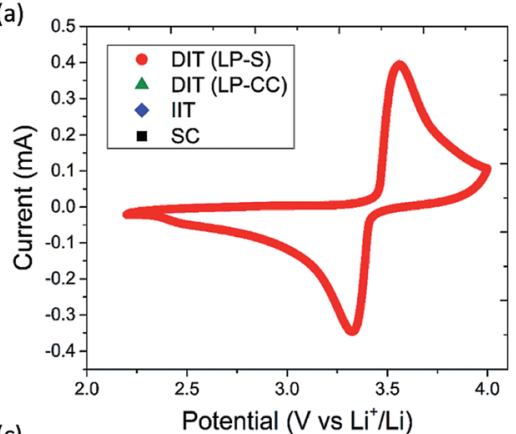

(c)

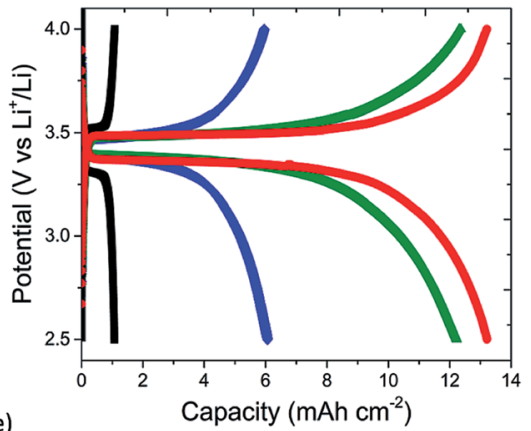

(e)

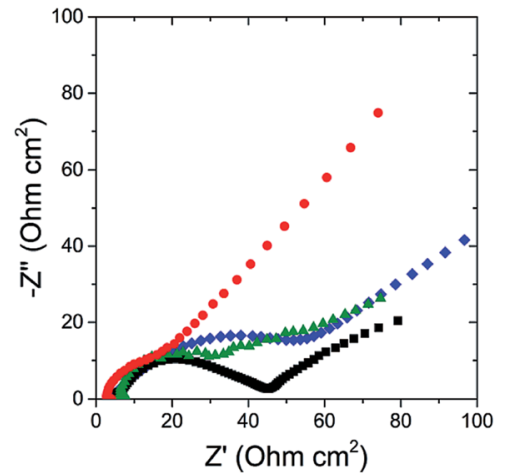

(b)

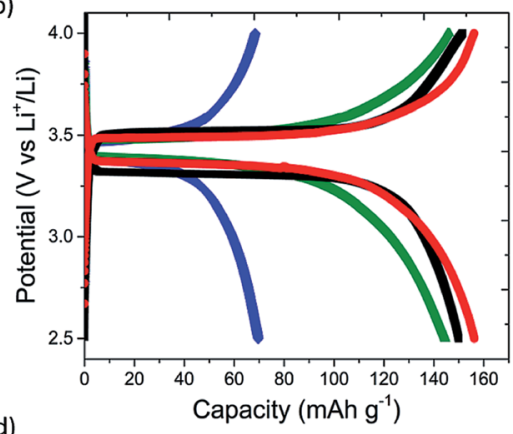

(d)

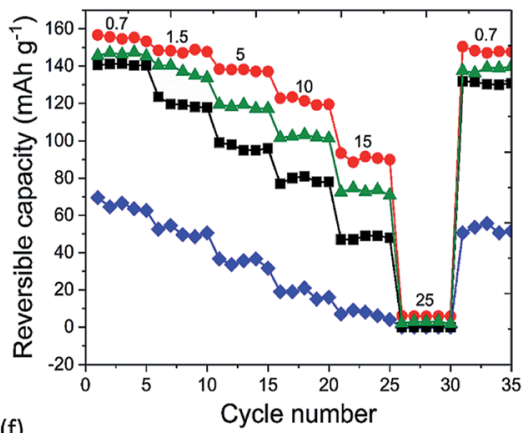

(f)

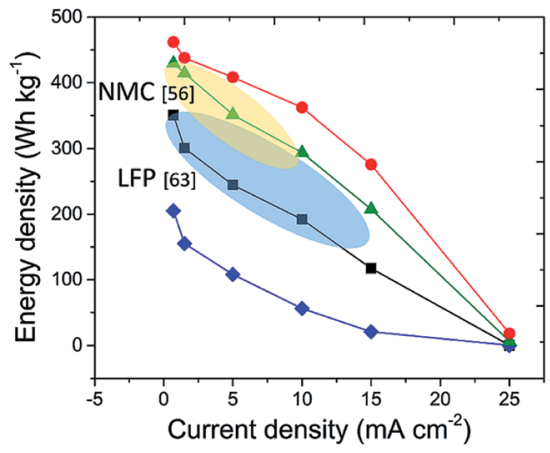

Fig. 4 (a) Cyclic voltammogram of the DIT (LP-S) electrode at a scan rate of $0.05 \mathrm{mV} \mathrm{s}^{-1}$; charge and discharge profiles of the DIT (LP-S), DIT (LPCC), IIT and SC electrodes at 0.05C showing (b) gravimetric capacities and (c) areal capacities; (d) reversible capacities of the four types of electrodes at different current densities (the annotations indicate current densities in $\mathrm{mA} \mathrm{cm}^{-2}$ ); (e) Nyquist plot of the corresponding four types of electrodes after rate capability testing (inset: cycling performance of the DIT (LP-S) electrode); and (f) energy densities at increasing current densities at the cell-stack level including inactive components.

charge and discharge profiles of the DIT (LP-S), DIT (LP-CC), IIT and SC electrodes at $0.05 \mathrm{C}$ in the second cycle. Given that the ice templated electrodes had significantly greater thickness and mass loading than the SC electrode, the corresponding applied current densities were $0.7 \mathrm{~mA} \mathrm{~cm}{ }^{-2}$ for the DIT and IIT electrodes and $0.06 \mathrm{~mA} \mathrm{~cm}^{-2}$ for the $\mathrm{SC}$ electrode. The reversible gravimetric capacity of the $900 \mu \mathrm{m}$ thick DIT (LP-S) electrode was $157 \mathrm{~mA} \mathrm{~h} \mathrm{~g}^{-1}$ based on the mass of active material, higher than the 151, 146 and $70 \mathrm{~mA} \mathrm{~h} \mathrm{~g}{ }^{-1}$ for the calendered SC, DIT (LP-CC) and IIT electrodes, respectively. The coulombic efficiency was 99 to $100 \%$ for all of the electrodes. Fig. 4(c) shows the same charge and discharge profiles with the corresponding areal capacities, showing reversible areal capacities of 13.2, 12.3, 6.1 and $1.1 \mathrm{~mA} \mathrm{~h} \mathrm{~cm}^{-2}$ for the DIT (LP-S), DIT (LP-CC), IIT and SC electrodes, respectively. The highly competitive areal capacities of the DIT (LP-S) electrode also compare favorably with the 1 to $3 \mathrm{~mA} \mathrm{~h} \mathrm{~cm}-2$ for standard SC electrodes of LFP and other cathode materials, e.g. $\mathrm{LiNi}_{0.8} \mathrm{Mn}_{0.1} \mathrm{Co}_{0.1} \mathrm{O}_{2} .{ }^{2,56}$

Fig. 4(d) shows the reversible areal capacities for the DIT (LPS), DIT (LP-CC), IIT and SC electrodes at each current density. The corresponding gravimetric capacities at each current are shown in Fig. S6. $\dagger$ The DIT (LP-S) electrode maintained the highest capacities, realising $7.9 \mathrm{~mA} \mathrm{~h} \mathrm{~cm}{ }^{-2}\left(94 \mathrm{~mA} \mathrm{~h} \mathrm{~g}^{-1}\right)$ at an ultra-high current density of $15 \mathrm{~mA} \mathrm{~cm}{ }^{-2}$. Above $15 \mathrm{~mA} \mathrm{~cm}{ }^{-2}$ ( $\sim 10$ times the current applied to the SC electrodes conventionally ${ }^{45,57}$ ), the difference between electrodes decreased markedly due to Li metal anode deterioration and the consequent very large polarizations. ${ }^{31}$ To match the performance of the ultra-thick DIT electrodes at the highest rates, new Li metal anode technology may be required. ${ }^{31}$ The competitive gravimetric and areal capacities and rate capability of the DIT (LP-S) electrode from 0.7 to $15 \mathrm{~mA} \mathrm{~cm}{ }^{-2}$ suggested that (i) the 
relatively dense, well-packed struts containing mostly primary particles (Fig. 2(e)) helped expose a relatively large electrolyte/ LFP contact area and supported efficient $\mathrm{Li}$ ion intercalation kinetics; (ii) since Li ion transport restriction becomes a dominant factor in capacity at fast charge and discharge rates, ${ }^{58}$ the aligned pore arrays in the DIT electrode microstructure more effectively maintained ion mobility and sustained capacity at high current densities despite the ultra-high thickness; and (iii) the electrode microstructure with lower $\varepsilon$, low $\tau_{z}$ and low $\tau_{x}$ near the separator and higher $\varepsilon$, even lower $\tau_{z}$ and high $\tau_{x}$ near the current collector performed better than the converse arrangement because Li ion mobility was promoted to be more uniform throughout the electrode.

Electrochemical impedance spectroscopy (EIS) was used to investigate the electrodes after the rate capability testing. Fig. 4(e) shows a Nyquist plot of the four types of electrodes where the intercept of a best-fit semi-circle to the data with the $Z^{\prime}$ axis indicates the combined electrical resistance of the electrode and ionic resistance of the electrolyte $R_{\mathrm{S}}$, which was estimated to be 4.0, 4.2, 7.8 and $8.1 \Omega \mathrm{cm}^{2}$ for the DIT (LP-S), DIT (LP-CC), IIT and SC electrodes, respectively. The lower $R_{\mathrm{S}}$ of the DIT electrodes than of the IIT and SC electrodes suggested that the anisotropic structure provided better electrical connectivity throughout the electrode, i.e. efficient electron transfer up and down the aligned struts. The diameter of the semi-circle of the Nyquist plot indicates the combined charge transfer resistance of the electrode and electrode/electrolyte interfacial resistance $R_{\mathrm{CT}}{ }^{59}$ and was estimated to be $21,32,40$ and $63 \Omega \mathrm{cm}^{2}$ for the DIT (LP-S), DIT (LP-CC), SC and IIT electrodes, respectively. The lowest $R_{\mathrm{CT}}$ and the highest gradient of the Nyquist tail for the DIT (LP-S) electrode confirmed faster effective Li ion mobility throughout the electrode. ${ }^{60}$ Fig. S7 $\dagger$ shows the reversible capacity of the DIT (LP-S) electrode after charge-discharge cycles at $0.1 \mathrm{C}$, with a capacity retention of $93 \%$ after 100 cycles, which was comparable with the $90-94 \%$ obtained for the SC electrodes. ${ }^{61}$

\section{Gravimetric and volumetric energy density at the cell-stack level}

Consideration is now given to the comparative performance of LIB cell-stacks for real applications if they were to be assembled using the different LFP-based cathodes investigated here. Four types of cell unit(s) of the same total volume were considered: (i) a LIB cell unit of a thick $(900 \mu \mathrm{m})$ DIT (LP-S) cathode, a separator, a Li counter electrode and standard current collectors (9 $\mu \mathrm{m}$ in thickness), (ii) a LIB cell unit of the same components except for a thick $(900 \mu \mathrm{m})$ DIT (LP-CC) cathode, (iii) a LIB cell unit of the same components except for a thick $(900 \mu \mathrm{m})$ IIT cathode, and (iv) a stack of LIB cell units of conventional calendered SC cathodes $(55 \mu \mathrm{m})$, separators, Li counter electrodes and current collectors. The method of estimating the energy densities is given in the ESI. $\uparrow$ The estimated volume and weight fractions of inactive components in each LIB cell-stack are shown in Table S1 in the ESI, $\uparrow$ and are consistent with those of commercial LIBs. ${ }^{62}$ The estimated energy densities are for comparative purposes because practical anodes with matching capacity for the ultra-thick DIT cathodes would be required, but nonetheless the data are robust for trend-wise comparison. ${ }^{\mathbf{1 4}}$ Fig. 4(f) shows their gravimetric energy densities against charge and discharge current densities at the cellstack level. A current density of $15 \mathrm{~mA} \mathrm{~cm} \mathrm{~cm}^{-2}$ corresponds to high power applications, e.g. the dynamic stress test for fast charging electric vehicles. ${ }^{4}$ Fig. $4(f)$ shows that the cell-stack containing the DIT (LP-S) cathode exhibits the highest energy densities among the four types of cell-stacks at all current densities including inactive components, and the achieved cellstack energy densities as well as the high current densities are also higher than those of the other cell-stacks containing commercial LFP electrodes ${ }^{63}$ and $\mathrm{LiNi}_{0.8} \mathrm{Mn}_{0.1} \mathrm{Co}_{0.1} \mathrm{O}_{2}$ electrodes. ${ }^{56}$

Table 1 summarizes the resulting gravimetric and volumetric energy densities at fast charge and discharge current densities of 5 and $15 \mathrm{~mA} \mathrm{~cm}^{-2}$. At a high current density of $5 \mathrm{~mA} \mathrm{~cm}$, the gravimetric energy density of the cell-stack containing the DIT (LP-S) electrode was $408 \mathrm{~W} \mathrm{~h} \mathrm{~kg}^{-1}$ (411 $\mathrm{W} \mathrm{h} \mathrm{cm}^{-3}$ ), higher than the 351,108 and $245 \mathrm{~W} \mathrm{~h} \mathrm{~kg}^{-1}\left(354,112\right.$ and $\left.388 \mathrm{~W} \mathrm{~h} \mathrm{~cm}^{-3}\right)$ for the same total cell-stack volume containing the DIT (LP-CC), IIT and SC electrodes. At an ultra-high current density of $15 \mathrm{~mA}$ $\mathrm{cm}^{-2}$, the gravimetric and volumetric energy densities of the cell-stack containing the DIT (LP-S) electrode were also maintained to be the highest amongst the four types. These results demonstrate that (i) the disadvantage of higher intrinsic porosity of a templated electrode was mitigated by its high thickness and its significant reduction in the fraction of inactive separators and current collectors required compared with conventional SC electrodes; (ii) the DIT (LP-S) electrode structure addressed the challenge of effective ion transport through thick electrodes and had the key advantage of maintaining high energy densities at high current densities; and (iii) the naturally formed gradient electrode structure (without other intricate steps of suppressing or promoting porosity) integrated into cells using the correct orientation was able to achieve high performance, demonstrating the scalability of the DIT process. Cost reduction is also predicted due to the reduced proportion of the relatively expensive inactive components. ${ }^{37}$

\section{Conclusions}

Ultra-thick LFP-based LIB cathodes with conical shaped pore channels extending through the electrode thickness were fabricated by directional ice templating. X-ray computed tomography of the electrodes and Li ion mobility simulations showed a graded and low tortuosity (through the electrode thickness) structure. The highest gravimetric and volumetric energy densities at the cell-stack level at ultra-high current densities were achieved when the cathode was oriented such that the low porosity, low tortuosity (through the electrode thickness) and low tortuosity (within the electrode plane) subdomain was adjacent to the separator, while the high porosity, even lower tortuosity (through the electrode thickness) and high tortuosity (within the electrode plane) sub-domain was adjacent to the current collector. The optimal performance was attributed to (i) improved uniformity of ion mobility 
throughout the thick electrode, (ii) low electrical resistance of the oriented struts of well-packed primary active materials with a large exposed electrolyte/LFP contact area, and (iii) a significantly reduced proportion of inactive components at the cellstack level. This work represents an important step towards the practicality of adopting novel battery manufacturing techniques to improve performance and opens up a promising prospect of optimally exploiting anisotropic and graded structures for energy storage and conversion devices that rely on directional mass transport.

\section{Experimental}

\section{Electrode fabrication}

LFP powders were provided by Hydro-Quebec. Electrode slurries were prepared by homogeneously mixing the LFP powders, Super P carbon black (Alfa Aesar) and sodium carboxymethyl cellulose (CMC, Sigma Aldrich) binder in a weight ratio of $90: 5: 5$ in deionized water. ${ }^{64-66}$ For fabricating the electrodes by DIT, the slurry was directionally frozen in a custom-made 3D printed acrylonitrile butadiene styrene (ABS) mould on a copper cold finger, one end of which was immersed in liquid nitrogen. Freezing at atmosphere pressure $(101 \mathrm{kPa})$ involved cooling from room temperature to $-10{ }^{\circ} \mathrm{C}$ until fully solid. The temperature was measured using a thermocouple inserted in the copper cold finger close to the interface with the electrode slurry. The free-standing frozen electrodes were extracted from the moulds and then freeze dried at $-5{ }^{\circ} \mathrm{C}$ under $1.5 \mathrm{~Pa}$ to sublime the ice. For comparison, electrodes were also fabricated by isotropic ice templating by placing the electrode slurry in a freezer and by conventional slurry casting followed by calendering using the same materials.

\section{Characterization}

The electrode weight was measured using a microbalance (Sartorius) with $0.01 \mathrm{mg}$ accuracy. The size distributions of the LFP primary and secondary particles were acquired through image processing software ImageJ. Briefly, a grey level threshold was first applied to the SEM images of the primary and secondary particles, a watershed algorithm was then applied to the binary images, and finally built-in particle analysis was used to quantify the equivalent diameter of each particle, and its distribution was plotted. The electrodes were investigated by Xray diffraction (XRD, Siemens D5000) with $\mathrm{Cu} \mathrm{K}_{\alpha}$ radiation $\lambda=$ $1.5 \AA$ A. Cross-sections of the electrodes were prepared using a Gatan precision etching coating system (PECS 685) with a $6 \mathrm{kV}$ beam for $2 \mathrm{~h}$. The electrode cross-sections were examined by scanning electron microscopy (SEM, Zeiss Merlin Analytical and JEOL 6500F) and energy dispersive X-ray (EDX) analysis (Zeiss Merlin Analytical). X-ray computed tomography (XCT) was performed on a Zeiss Xradia; each XCT scan comprises 3142 2D radiographic projections. All XCT scans were reconstructed into a 3D volume using filtered back projection and beam hardening correction algorithms embedded in a Scout-and-Scan Control System Reconstructor (Zeiss). The reconstructed image volumes were post-processed and quantified using Avizo 9.7.0 (Thermo
Fisher Scientific, USA). A 3D median filter with a kernel of $3 \times 3$ $\times 3$ was applied to all image volumes for noise removal. ${ }^{67}$ The filtered image volumes were then segmented using the Otsu threshold, ${ }^{68}$ resulting in binarized image volumes for analysis. Directional tortuosity was estimated using the TauFactor code in MatLab. ${ }^{51}$ All electrodes were investigated following assembly in an Ar filled glovebox with $\mathrm{Li}$ foil as the counter electrode and an electrolyte of $1 \mathrm{M} \mathrm{LiPF}_{6}$ dissolved in a mixed solvent of ethylene carbonate (EC) and diethyl carbonate (DEC) in $1: 1 \mathrm{v} / \mathrm{v}$. The cells were galvanostatically charged and discharged between 2.5 and $4.0 \mathrm{~V}$ vs. $\mathrm{Li} / \mathrm{Li}^{+}$at different currents using an Arbin BT-G-25 cycler. Cyclic voltammetry was performed between 2.1 and $4.0 \mathrm{~V}$ at $0.05 \mathrm{mV} \mathrm{s}^{-1}$ and electrochemical impedance spectroscopy (EIS) was performed in the $2 \times 10^{6}$ to $0.01 \mathrm{~Hz}$ frequency range at room temperature using a Gamry Reference 600/EIS300 potentiostat/galvanostat. Sixty cells from different DIT experiments were measured to verify reproducibility of capacities and gave a variation of $\pm 8 \%$.

\section{Conflicts of interest}

The author(s) declare no competing financial interests.

\section{Acknowledgements}

This work was supported by EPSRC grants EP/S001239/1 "Novel Manufacturing Approaches to Next Generation Batteries" and EP/M009521/1 "Enabling Next generation Li batteries". The authors acknowledge the financial support from the Henry Royce Institute (through UK Engineering and Physical Sciences Research Council grant EP/R010145/1) for capital equipment. The use of facilities funded by EPSRC Grant EP/M02833X/1 University of Oxford: experimental equipment upgrade is gratefully acknowledged. The authors thank Prof. Peter G. Bruce for the precision etching and coating system (PECS) sample preparation and Prof. Mauro Pasta for glovebox access.

\section{References}

1 J.-Y. Hwang, S.-J. Park, C. S. Yoon and Y.-K. Sun, Customizing a Li-metal battery that survives practical operating conditions for electric vehicle applications, Energy Environ. Sci., 2019, 12, 2174-2184.

2 J. Liu, Z. Bao, Y. Cui, E. J. Dufek, J. B. Goodenough, P. Khalifah, Q. Li, B. Y. Liaw, P. Liu, A. Manthiram, Y. S. Meng, V. R. Subramanian, M. F. Toney, V. V. Viswanathan, M. S. Whittingham, J. Xiao, W. Xu, J. Yang, X.-Q. Yang and J.-G. Zhang, Pathways for practical high-energy long-cycling lithium metal batteries, Nat. Energy, 2019, 4, 180-186.

$3 \mathrm{~J}$. M. Tarascon and M. Armand, Issues and challenges facing rechargeable lithium batteries, Nature, 2001, 414, 359-367.

4 J. S. Sander, R. M. Erb, L. Li, A. Gurijala and Y.-M. Chiang, High-performance battery electrodes via magnetic templating, Nat. Energy, 2016, 1, 1-7.

5 D. Gordon, M. Y. Wu, A. Ramanujapuram, J. Benson, J. T. Lee, A. Magasinski, N. Nitta, C. Huang and G. Yushin, 
Enhancing cycle stability of lithium iron phosphate in aqueous electrolytes by increasing electrolyte molarity, $A d v$. Energy Mater., 2016, 6, 1501805.

6 H. Zheng, J. Li, X. Song, G. Liu and V. S. Battaglia, A comprehensive understanding of electrode thickness effects on the electrochemical performances of Li-ion battery cathodes, Electrochim. Acta, 2012, 71, 258-265.

7 M. Singh, J. Kaiser and H. Hahna, Thick electrodes for high energy lithium ion batteries, J. Electrochem. Soc., 2015, 162(7), A1196-A1201.

8 C. Chen, Y. Zhang, Y. Li, Y. Kuang, J. Song, W. Luo, Y. Wang, Y. Yao, G. Pastel, J. Xie and L. Hu, Highly conductive, lightweight, low-tortuosity carbon frameworks as ultrathick 3D current collectors, Adv. Energy Mater., 2017, 7, 1700595.

9 K. Evanoff, J. Khan, J. A. A. Balandin, A. Magasinski, W. J. Ready, T. F. Fuller and G. Yushin, Towards ultrathick battery electrodes: aligned carbon nanotube-enabled architecture, Adv. Mater., 2012, 24(4), 533-537.

10 M. Ebner, D.-W. Chung, R. E. Garcia and V. Wood, Tortuosity anisotropy in lithium-ion battery electrodes, Adv. Energy Mater., 2014, 4, 1301278.

11 J. Newman and K. E. Thomas-Alyea, Electrochemical Systems, John Wiley \& Sons, Inc, 2004.

12 M. Doyle and J. Newman, Analysis of capacity-rate data for lithium batteries using simplified models of the discharge process, J. Appl. Electrochem., 1997, 27(7), 846-856.

13 N. Balke, S. Jesse, A. N. Morozovska, E. Eliseev, D. W. Chung, Y. Kim, L. Adamczyk, R. E. Garcia, N. Dudney and S. V. Kalinin, Nanoscale mapping of ion diffusion in a lithium-ion battery cathode, Nat. Nanotechnol., 2010, 5, 749-754.

14 F. Wu and G. Yushin, Conversion cathodes for rechargeable lithium and lithium-ion batteries, Energy Environ. Sci., 2017, 10, 435-459.

15 T. Marks, S. Trussler, A. J. Smith, D. Xiong and J. R. Dahn, A guide to Li-ion coin-cell electrode making for academic researchers, J. Electrochem. Soc., 2011, 158(1), A51-A57.

16 S. Oro, K. Urita and I. Moriguchi, Nanospace-controlled $\mathrm{SnO}_{2} /$ nanoporous carbon composite as a high-performance anode for sodium ion batteries, Chem. Lett., 2017, 46, 502505.

17 S. Oro, K. Urita and I. Moriguchi, Nanospace control of $\mathrm{SnO}_{2}$ nanocrystallites-embedded nanoporous carbon for reversible electrochemical charge-discharge reactions, $J$. Phys. Chem. C, 2016, 120, 25717-25724.

18 Y. Zhang, Y. Lu, S. Feng, D. Liu, Z. Ma and S. Wang, On-site evolution of ultrafine $\mathrm{ZnO}$ nanoparticles from hollow metalorganic frameworks for advanced lithium ion battery anodes, J. Mater. Chem. A, 2017, 5, 22512-22518.

19 H. Liu, L. Tao, Y. Zhang, C. Xie, P. Zhou, H. Liu, R. Chen and S. Wang, Bridging covalently functionalized black phosphorus on graphene for high-performance sodium-ion battery, ACS Appl. Mater. Interfaces, 2017, 9(42), 3684936856.

20 H. Liu, Y. Zou, L. Tao, Z. Ma, D. Liu, P. Zhou, H. Liu and S. Wang, Sandwiched thin-film anode of chemically bonded black phosphorus/graphene hybrid for lithium-ion battery, Small, 2017, 13, 1700758.

21 Z. Ma, L. Tao, D. Liu, Z. Li, Y. Zhang, Z. Liu, H. Liu, R. Chen, J. Huo and S. Wang, Ultrafine nano-sulfur particles anchored on in situ exfoliated graphene for lithium-sulfur batteries, $J$. Mater. Chem. A, 2017, 5, 9412-9417.

22 Z. Li, H. B. Wu and X. W. D. Lou, Rational designs and engineering of hollow micro-/nanostructures as sulfur hosts for advanced lithium-sulfur batteries, Energy Environ. Sci., 2016, 9, 3061-3070.

23 S. L. Zhang, B. Y. Guan, H. B. Wu and X. W. D. Lou, Metalorganic framework-assisted synthesis of compact $\mathrm{Fe}_{2} \mathrm{O}_{3}$ nanotubes in $\mathrm{Co}_{3} \mathrm{O}_{4}$ host with enhanced lithium storage properties, Nano-Micro Lett., 2018, 10, 44.

24 Y. Lu, J. Nai and X. W. D. Lou, Formation of $\mathrm{NiCo}_{2} \mathrm{~V}_{2} \mathrm{O}_{8}$ yolkdouble shell spheres with enhanced lithium storage properties, Angew. Chem., Int. Ed., 2018, 57, 2899-2903.

25 Y. Lu, L. Yu, M. Wu, Y. Wang and X. W. D. Lou, Construction of complex $\mathrm{Co}_{3} \mathrm{O}_{4} @ \mathrm{Co}_{3} \mathrm{~V}_{2} \mathrm{O}_{8}$ hollow structures from metalorganic frameworks with enhanced lithium storage properties, Adv. Mater., 2018, 30, 1702875.

26 C. Huang, N. P. Young, J. Zhang, H. J. Snaith and P. S. Grant, A two layer electrode structure for improved Li ion diffusion and volumetric capacity in Li ion batteries, Nano Energy, 2017, 31, 377-385.

27 C. Huang, J. Zhang, H. J. Snaith and P. S. Grant, Engineering the membrane/electrode interface to improve the performance of solid-state supercapacitors, ACS Appl. Mater. Interfaces, 2016, 8, 20756-20765.

28 C. Huang, N. Grobert, A. A. R. Watt, C. Johnston, A. Crossley, N. P. Young and P. S. Grant, Layer-by-layer spray deposition and unzipping of single-wall carbon nanotube-based thin film electrodes for electrochemical capacitors, Carbon, 2013, 61, 525-536.

29 C.-J. Bae, C. K. Erdonmez, J. W. Halloran and Y.-M. Chiang, Design of battery electrodes with dual-scale porosity to minimize tortuosity and maximize performance, $A d v$. Mater., 2013, 25, 1254-1258.

30 L. Li, R. M. Erb, J. Wang, J. Wang and Y.-M. Chiang, Fabrication of low-tortuosity ultrahigh-area-capacity battery electrodes through magnetic alignment of emulsion-based slurries, Adv. Energy Mater., 2019, 9, 1802472.

31 L.-L. Lu, Y.-Y. Lu, Z.-J. Xiao, T.-W. Zhang, F. Zhou, T. Ma, Y. Ni, H.-B. Yao, S.-H. Yu and Y. Cui, Wood-inspired highperformance ultrathick bulk battery electrodes, Adv. Mater., 2018, 1706745.

32 S. Behr, R. Amin, Y. M. Chiang and A. P. Tomsia, Highly structured, additive free lithium-ion cathodes by freezecasting technology, Ceram. Forum Int., 2015, 92, E39-E43.

33 S. K. Wilke and D. C. Dunand, Structural evolution of directionally freeze-cast iron foams during oxidation/ reduction cycles, Acta Mater., 2019, 162, 90-102.

34 A. Kajdos, A. Kvit, F. Jones, J. Jagiello and G. Yushin, Tailoring the pore alignment for rapid ion transport in microporous carbons, JACS, 2010, 132, 3252-3253.

35 J. Ni, W. Wang, C. Wu, H. Liang, J. Maier, Y. Yu and L. Li, Highly reversible and durable $\mathrm{Na}$ storage in niobium 
pentoxide through optimizing structure, composition, and nanoarchitecture, Adv. Mater., 2017, 29, 1605607.

36 J. Ni, S. Fu, Y. Yuan, L. Ma, Y. Jiang, L. Li and J. Lu, Boosting sodium storage in $\mathrm{TiO}_{2}$ nanotube arrays through surface phosphorylation, Adv. Mater., 2018, 30, 1704337.

37 C. Huang and P. S. Grant, Coral-like directional porosity lithium ion battery cathodes by ice templating, J. Mater. Chem. A, 2018, 6, 14689.

38 A. Nyten, A. Abouimrane, M. Armand, T. Gustafsson and J. O. Thomas, Electrochemical performance of $\mathrm{Li}_{2} \mathrm{FeSiO}_{4}$ as a new Li-battery cathode material, Electrochem. Commun., 2005, 7, 156-160.

$39 \mathrm{~K}$. Araki and J. W. Halloran, New freeze-casting technique for ceramics with sublimable vehicles, JACS, 2004, 87(10), 18591863.

40 C.-Y. Hsieh, S.-P. Tsai, M.-H. Ho, D.-M. Wang, C.-E. Liu, C.-H. Hsieh, H.-C. Tseng and H.-J. Hsieh, Analysis of freeze-gelation and cross-linking processes for preparing porous chitosan scaffolds, Carbohydr. Polym., 2007, 67, 124-132.

41 X. Wang, A. J. Schultz and Y. Halpern, Kinetics of methane hydrate formation from polycrystalline deuterated ice, $J$. Phys. Chem. A, 2002, 106(32), 7304-7309.

42 S. W. Sofie, Fabrication of functionally graded and aligned porosity in thin ceramic substrates with the novel freezetape-casting process, J. Am. Ceram. Soc., 2007, 90(7), 20242031.

43 A. Lasalle, C. Guizard, E. Maire, J. Adrien and S. Deville, Particle redistribution and structural defect development during ice templating, Acta Mater., 2012, 60, 4594-4603.

44 Y. Liu, X.-Y. Yu, Y. Fang, X. Zhu, J. Bao, X. Zhou and X. W. D. Lou, Confining $\mathrm{SnS}_{2}$ ultrathin nanosheets in hollow carbon nanostructures for efficient capacitive sodium storage, Joule, 2018, 2, 725-735.

45 T. Huria, G. Ludovici and G. Lutzemberger, State of charge estimation of high power lithium iron phosphate cells, $J$. Power Sources, 2014, 249, 92-102.

46 S. J. Cooper, D. S. Eastwood, J. Gelb, G. Damblanc, D. J. L. Brett, R. S. Bradley, P. J. Withers, P. D. Lee, A. J. Marquis, N. P. Brandon and P. R. Shearing, Image based modelling of microstructural heterogeneity in $\mathrm{LiFePO}_{4}$ electrodes for Li-ion batteries, J. Power Sources, 2014, 247, 1033-1039.

47 F. A. L. Dullien, Porous Media: Fluid Transport and Pore Structure, Academic Press, San Diego, 1992.

48 A. C. Forse, J. M. Griffin, C. Merlet, J. Carretero-Gonzalez, A.-R. O. Raji, N. M. Trease and C. P. Grey, Direct observation of ion dynamics in supercapacitor electrodes using in situ diffusion NMR spectroscopy, Nat. Energy, 2017, 2, 16216.

49 O. Pecher, J. Carretero-González, K. J. Griffith and C. P. Grey, Materials' methods: NMR in battery research, Chem. Mater., 2017, 29, 213-242.

50 A. Kraytsberg and Y. Ein-Eli, Conveying Advanced Li-ion Battery Materials into Practice, The Impact of Electrode Slurry Preparation Skills, Adv. Energy Mater., 2016, 6, 1600655.
51 S. J. Cooper, A. Bertei, P. R. Shearing, J. A. Kilner and N. P. Brandon, TauFactor: An open-source application for calculating tortuosity factors from tomographic data, SoftwareX, 2016, 5, 203-210.

52 S. R. Daemi, X. Lu, D. Sykes, J. Behnsen, C. Tan, A. PalaciosPadros, J. Cookson, E. Petrucco, P. J. Withers, D. J. L. Brett and P. R. Shearing, 4D visualisation of in situ nanocompression of Li-ion cathode materials to mimic early stage calendering, Mater. Horiz., 2019, 6, 612-617.

53 C. Tan, S. R. Daemi, O. O. Taiwo, T. M. M. Heenan, D. J. L. Brett and P. R. Shearing, Evolution of electrochemical cell designs for in situ and operando 3D characterization, Materials, 2018, 11, 2157.

54 K. L. Scotti, L. G. Kearney, J. Burns, M. Ocana, L. Duros, A. Shelhamer and D. C. Dunand, The effect of solidification direction with respect to gravity on icetemplated $\mathrm{TiO}_{2}$ microstructures, J. Eur. Ceram. Soc., 2019, 39, 3180-3193.

55 M. Sato, S. Tajimi, H. Okawa, K. Uematsu and K. Toda, Preparation of iron phosphate cathode material of $\mathrm{Li}_{3} \mathrm{Fe}_{2}\left(\mathrm{PO}_{4}\right)_{3}$ by hydrothermal reaction and thermal decomposition processes, Solid State Ionics, 2002, 152-153, 247-251.

56 D. Andre, S.-J. Kim, P. Lamp, S. F. Lux, F. Maglia, O. Paschos and B. Stiaszny, Future generations of cathode materials: an automotive industry perspective, J. Mater. Chem. A, 2015, 3, 6709-6732.

57 J. B. Habedank, L. Kraft, A. Rheinfeld, C. Krezdorn, A. Jossen and M. F. Zaeh, Increasing the discharge rate capability of lithium-ion cells with laser-structured graphite anodes: modeling and simulation, J. Electrochem. Soc., 2018, 165(7), A1563-A1573.

58 K. G. Gallagher, S. E. Trask, C. Bauer, T. Woehrle, S. F. Lux, M. Tschech, P. Lamp, B. J. Polzin, S. Ha, B. Long, Q. Wu, W. Lu, D. W. Dees and A. N. Jansen, Optimizing areal capacities through understanding the limitations of lithium-ion electrodes, J. Electrochem. Soc., 2015, 163, A138.

59 B. Shen, P. Zuo, P. Fan, J. Yang, G. Yin, Y. Ma, X. Cheng, C. Du and Y. Gao, Improved electrochemical performance of $\mathrm{NaAlO}_{2}$-coated $\mathrm{LiCoO}_{2}$ for lithium-ion batteries, J. Solid State Electrochem., 2017, 21, 1195-1201.

60 N. Schweikert, R. Heinzmann, A. Eichhöfer, H. Hahn and S. Indris, Electrochemical impedance spectroscopy of $\mathrm{Li}_{4} \mathrm{Ti}_{5} \mathrm{O}_{12}$ and $\mathrm{LiCoO}_{2}$ based half-cells and $\mathrm{Li}_{4} \mathrm{Ti}_{5} \mathrm{O}_{12} /$ $\mathrm{LiCoO}_{2}$ cells: Internal interfaces and influence of state-ofcharge and cycle number, Solid State Ionics, 2012, 226, 15-23.

61 D. Jean-Christophe, F. Barray, C. Gagnon, D. Clément, P. Hovington, H. Demers, A. Guerfi and K. Zaghib, Amphiphilic latex as a water-based binder for $\mathrm{LiFePO}_{4}$ cathode, J. Power Sources, 2019, 415, 172-178.

62 D. L. Wood III, J. Li and C. Daniel, Prospects for reducing the processing cost of lithium ion batteries, J. Power Sources, 2015, 275, 234-242.

63 N. Nitta, F. Wu, J. T. Lee and G. Yushin, Li-ion battery materials: present and future, Mater. Today, 2015, 18(5), 252-264. 
64 S. H. Lee, C. Huang and P. S. Grant, Layer-by-layer printing of multi-layered heterostructures using $\mathrm{Li}_{4} \mathrm{Ti}_{5} \mathrm{O}_{12}$ and $\mathrm{Si}$ for high power Li-ion storage, Nano Energy, 2019, 61, 96-103.

65 S. H. Lee, C. Huang, C. Johnston and P. S. Grant, Spray printing and optimization of anodes and cathodes for high performance Li-ion batteries, Electrochim. Acta, 2018, 292, 546-557.

66 S. H. Lee, A. Mahadevegowda, C. Huang, J. D. Evans and P. S. Grant, Spray printing of self-assembled porous structures for high power battery electrodes, J. Mater. Chem. A, 2018, 6, 13133-13141.

67 C. L. A. Leung, R. Tosi, E. Muzangaza, S. Nonni, P. J. Withers and P. D. Lee, Effect of preheating on the thermal, microstructural and mechanical properties of selective electron beam melted Ti-6Al-4V components, Mater. Des., 2019, 174, 107792.

68 N. Otsu, A threshold selection method from gray-level histograms, IEEE Transactions on Systems, Man, and Cybernetics, 1979, 9, 62-66. 